\title{
REF Select: Expert System Software for Selecting Restriction Endonucleases for Restriction Endonuclease Fingerprinting
}

BioTechniques 27:1188-1201 (December 1999)

\author{
William A. Scaringe, Dongzhou Liao, Qiang \\ Liu and Steve S. Sommer \\ City of Hope National Medical Center, \\ Duarte, CA, USA
}

\begin{abstract}
REF Select, expert system software, has been developed to assist in the selection of optimal restriction endonucleases for restriction endonuclease fingerprinting ( $R E F)$, a method for rapid and sensitive mutation screening of long DNA segments $(1-2 \mathrm{~kb})$. The REF method typically involves six separate digestions with up to two restriction endnonucleases used in each digestion. If done manually, performing a comprehensive review of the large number of possible sets of restriction endonucleases that could be used (over $10^{19}$ in the example presented here) and making an optimal choice is not feasible. Furthermore, the typical nonoptimal manual selection takes approximately 8 h by someone experienced with REF. REF Select enables a comprehensive review of the possible sets and a consistent, objective and fast selection of an optimal set by using a two-step strategy: the selection of sets that meet specific constraints, which is followed by a ranking of those sets by an optimality score. Based on our experience with REF, we chose default selection and ranking parameters to help the user get started quickly. These parameters form a knowledge base that can be customized and then saved by the user. In conclusion, REF Select facilitates the general application of REF by serving as an expert system for the selection of optimal restriction endonucleases. We demonstrated REF Select using an example segment from the human $\mathrm{p} 53$ gene.
\end{abstract}

\section{INTRODUCTION}

Restriction endonuclease fingerprinting (REF) has been demonstrated as a rapid method for screening long segments of DNA for sequence variants $(1-4,13,14)$ with high sensitivity $(8,10)$. REF involves the separate digestions of a PCR-amplified DNA segment, typically with six groups of one or two restriction endonucleases (Figure 1). The test segment could be one contiguous segment of DNA or could consist of several smaller segments combined for digestion. The fragments from all digestions are combined into one lane of a non-denaturing gel and electrophoresed. Variants may be detected by an altered band pattern that is the result of one or both of two components: an informative restriction component (10) resulting from the loss or gain of a restriction site and/or an informative single-strand conformation polymorphism (SSCP) component $(5-7,9,11)$. Because the test segment is cut at a variety of sites and into a variety of fragment sizes by several groups of restriction endonucleases, the sensitivity of both the restriction component and the SSCP component are increased.

We developed the REF Select software to assist in the selection of restriction endonucleases that maximize the detection sensitivity. REF Select enables the application of consistent and objective criteria to the selection of an optimum set of groups of restriction endonucleases from many possible sets. Before the availability of REF Select, it took approximately eight hours for someone experienced with REF methodology to manually select appropriate but nonoptimal restriction endonucleases. With REF Select, even an inexperienced user without extensive knowledge of restriction endonucleases can make an optimal selection in approximately ten minutes.

\section{MATERIALS AND METHODS}

REF Select conforms to the American National Standards Institute (ANSI) standard for the $\mathrm{C}$ programming language and is available on the Internet at http://www.cityofhope. org/molgen/bioinformatics.asp. This Web site can also be accessed through a link from the Software Library on the BioTechniques Web site (www.biotechniques.com). Input is provided by a simple interactive text-based user interface and American Standard Code for Information Interchange (ASCII) text files. Default parameter values and online help are provided. The various parameters can be modified and then saved to a parameter file for later reuse. For advanced users, command line options are provided for batch processing.

REF Select is designed to work in concert with the GCG Wisconsin Package (Genetics Computer Group, Madison, 
WI, USA; http://www.gcg.com); it uses the output of GCG Mapsort and the graphics capabilities of GCG Figure. By using GCG Mapsort to get the set of restriction endonucleases that cut the DNA segment and the corresponding cut sites, REF Select makes indirect use of REBASE (http://rebase. neb.com) (12), a restriction endonuclease database. REF Select can also read the restriction sites from two programs that are available from the Internet without cost: TACG (part of the Biology Workbench at http://biology.ncsa.uiuc.edu) and Webcutter 2 (http://www.firstmarket.com/cutter/cut2.html), which is useful for those who do not have access to GCG.

\section{RESULTS AND DISCUSSION}

REF Select uses a two-step strategy of selection that is followed by ranking using an optimality scoring algorithm (Figure 2). The selection step eliminates restriction endonucleases, groups and sets that do not meet the user-specified selection constraints (Table 1) and substantially reduces the burden of the ranking step. The first phase of the selection step is the application of constraints on the individual restriction endonucleases: they must cut the DNA segment at least once, and they must be available and desirable, as defined by the user; typically, it is desired that they be substantially free

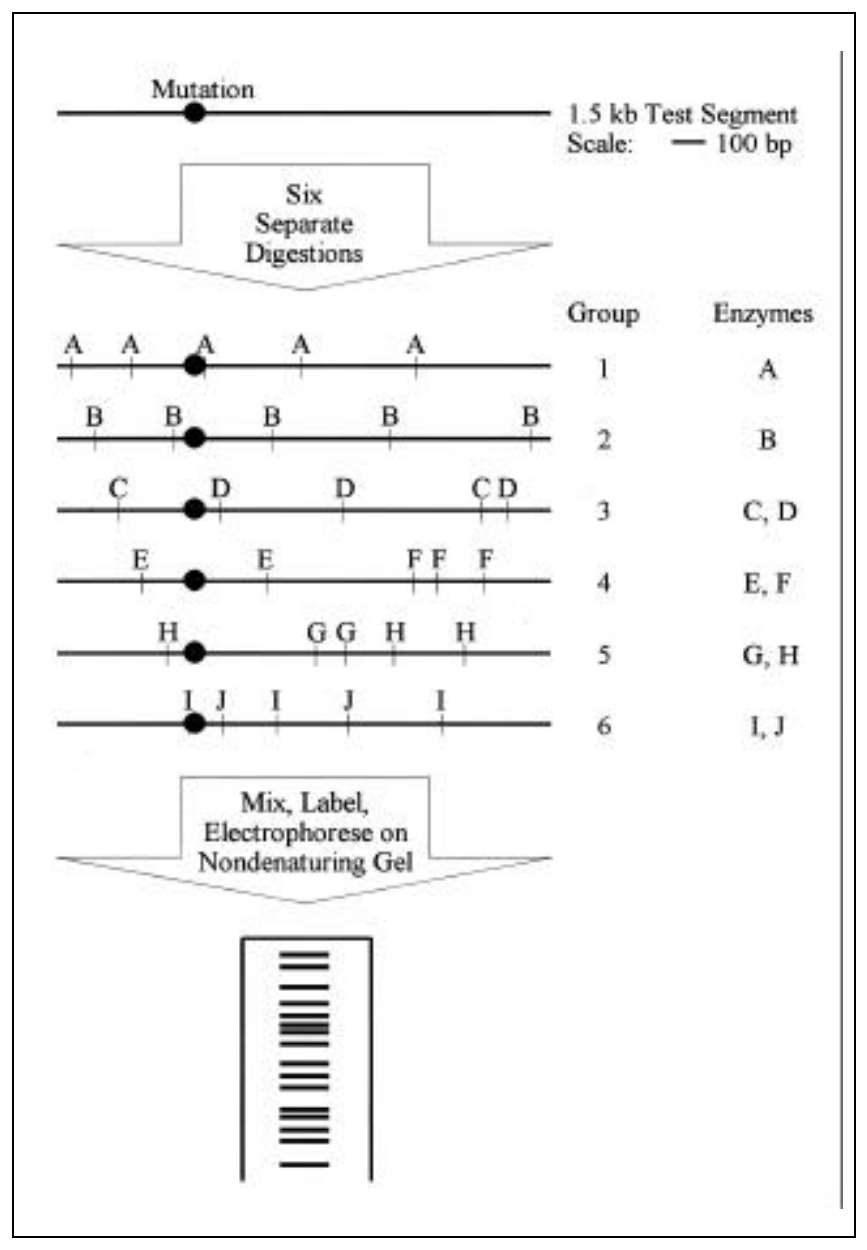

of star activity, free of dramatic variation in cleavage efficiency at different sites and not prohibitively expensive, etc. If only this first phase of constraints were applied, the number of possible sets would usually be unmanageable even with a computer, as demonstrated by the $p 53$ example presented in the Appendix, in which there are over $10^{19}$ possible sets of six groups of one or two of the 105 available and desirable restriction endonucleases that cut the segment. A dramatic reduction in the number of sets that must be ranked is achieved by applying the group (second phase) and set (third phase) selection constraints. In the $p 53$ example, only 52678 sets remain to be ranked after all the selection constraints are applied, a number that can be quickly ranked by a modern desktop computer.

\section{Input}

The required file input to REF Select is a Mapsort file generated by GCG Mapsort (see the example command sequence in the Appendix). This file lists all the restriction endonucleases that cut the DNA segment and the locations of the cut sites (two alternative restriction site input file formats are also supported; see Materials and Methods and the online help). An optional input file, called the restriction endonuclease property file, serves as a user database of restriction endonucleases (see the example in the Appendix). In addition to limiting the restriction endonucleases to those marked as available and desirable, the property file provides information (optimum digestion temperature and buffer activity levels) that REF Select uses to ensure compatibility between restriction endonucleases used together in a digestion group.

The input parameters are categorized as group selection constraints, set selection constraints, scoring algorithm parameters, output parameters and program mode. REF Select prompts the user for all needed input parameters in a logical sequence (see the example in the Appendix). The default values for the selection constraints are based on experience with REF and form a knowledge base (Table 1) that helps the user get started quickly.

\section{Features}

Automatic parameter adjustment. Adjustments to the default selection constraints may be needed to get optimal output for a particular DNA segment. REF Select includes an optional automatic parameter adjustment mode that aids the user when the initial input parameters result in no selected sets or too many selected sets (i.e., too many to process on a reasonable computer in a reasonable amount of time). Because of boundary effects, the number of selected sets may

Figure 1. Schematic of REF. A hypothetical $1.5 \mathrm{~kb}$ test segment with a point mutation is digested separately by 6 groups of 1 or 2 restriction endonucleases as shown. A total of 10 hypothetical restriction endonucleases (A-J) are used to generate 72 fragments ( 36 sense and 36 antisense) ranging in size from $50-450 \mathrm{bp}$. Group 1 fragment sizes in bases from left to right are: 50, 170, 230, 290, 350 and 410; Group 2: 120, 240, 300, 360, 420 and 60; Group 3: 190, 310, 370, 430, 70 and 130; Group 4: 260, 380, 440, 80, 140 and 200; Group 5: 330, 450, 90, 150, 210 and 270; and Group 6: 400, 100, 160, 220, 280 and 340. 
Table 1. Selection Constraints

\begin{tabular}{|c|c|c|c|c|}
\hline & No. & Constraint & Default & Automatic Adjustment \\
\hline \multirow{4}{*}{ 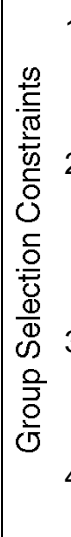 } & & $\begin{array}{l}\text { Maximum Digestion Temperature } \\
\text { Difference (compatibility constraint } \\
\text { for groups of two enzymes) }\end{array}$ & $5^{\circ} \mathrm{C}$ & No \\
\hline & & $\begin{array}{l}\text { Minimum Buffer Activity Levels } \\
\text { (compatibility constraint for groups } \\
\text { of two enzymes) }\end{array}$ & $\begin{array}{l}\text { Both enzymes must have } \\
\text { activities of } 100 \text { in at least } \\
\text { one available buffer }\end{array}$ & $\begin{array}{l}\text { Adjusted in increments of } 25 \text { down to a } \\
\text { minimum of } 50 \text { if too few groups selected or } \\
\text { up to a maximum of } 100 \text { if too many groups } \\
\text { selected }\end{array}$ \\
\hline & & Fragment Size Range & $50-500 \mathrm{bp}$ & $\begin{array}{l}\text { Maximum increased in increments of } 10 \% \\
\text { if too few groups selected and minimum } \\
\text { buffer activity levels at } 50 \text { or lower }\end{array}$ \\
\hline & 4 & Range of No. of Groups to Select & $25-125$ & $\begin{array}{l}\text { Specifies when adjustment of other } \\
\text { constraints is needed }\end{array}$ \\
\hline \multirow{5}{*}{ 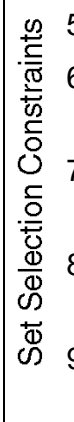 } & & No. of Groups in a Set & 6 & No \\
\hline & & $\begin{array}{l}\text { Total No. of Fragments (sense and } \\
\text { antisense fragments from all groups) }\end{array}$ & $100(+10 \%,-20 \%)$ & No \\
\hline & & $\begin{array}{l}\text { 5' Maximum End Fragment } \\
\text { Size }\end{array}$ & $\begin{array}{l}\text { Size over } 200 \text { bp limited } \\
\text { to no more than } 3 \text { groups }\end{array}$ & $\begin{array}{l}\text { Oversize limit increased in increments of } \\
\text { one if no valid sets found }\end{array}$ \\
\hline & & 3’ Maximum End Fragment Size & $\begin{array}{l}\text { Size over } 200 \text { bp limited to no } \\
\text { more than } 3 \text { groups }\end{array}$ & $\begin{array}{l}\text { Oversize limit increased in increments of } \\
\text { one if no valid sets found }\end{array}$ \\
\hline & 9 & $\begin{array}{l}\text { Maximum No. of Common Cuts } \\
\text { (between any two groups) }\end{array}$ & $\begin{array}{l}\text { No more than } 2 \text { cuts separated } \\
\text { by } 4 \text { bp or less }\end{array}$ & No \\
\hline
\end{tabular}

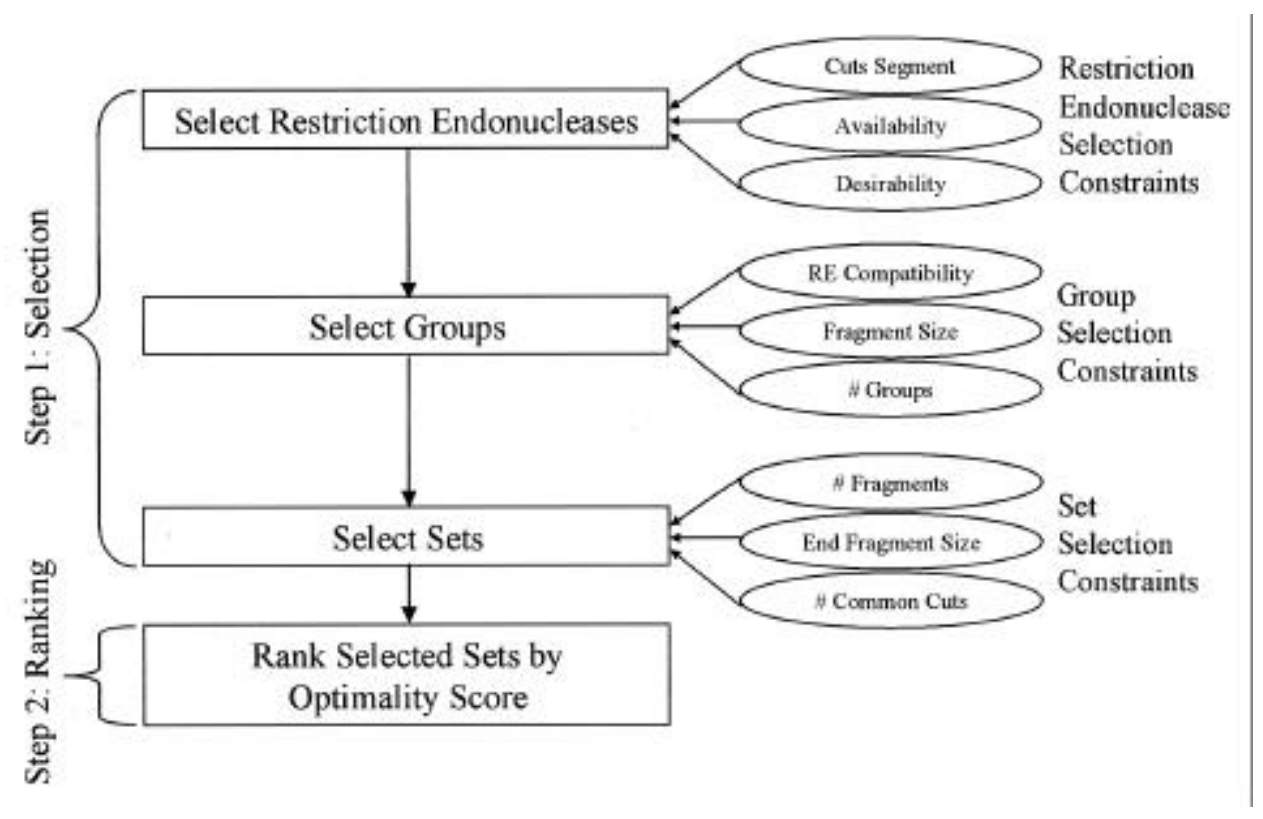

Figure 2. REF Select two-step strategy of selection followed by ranking. Application of the selection constraints is subdivided into three sequential selection phases: select restriction endonucleases, select groups and select sets. A group consists of either one or two restriction endonucleases. REF Select first considers all possible groups with one restriction endonuclease and then all with two restriction endonucleases. When groups with two restriction endonucleases are considered, compatibility constraints are applied. A set is a combination of the specified number of groups (six by default). The selected sets are ranked by a theoretical optimality score (see Table 1 for the selection constraints and Equation 1 for the scoring formula). 
change dramatically with small parameter changes. For example, most available restriction endonucleases may be excluded by a particular maximum fragment-size selection constraint that results in too few selected sets. If this size constraint is relaxed by only a small percentage, however, more restriction endonucleases may become acceptable, which results in a dramatic combinatorial increase in set possibilities. Automatic parameter adjustment helps avoid the tedium of manually homing in on the desired side of such a boundary by automatically making incremental parameter adjustments (Table 1) and iterating until the number of selected sets is in the desired range. Such adjustments may not always result in convergence, so the number of adjustments is limited.

Multiple passes. Although REF Select can rank thousands of sets that meet the selection constraints, the user will typically specify that only a few at the top end of the ranking be output (three by default). The difference between these sets

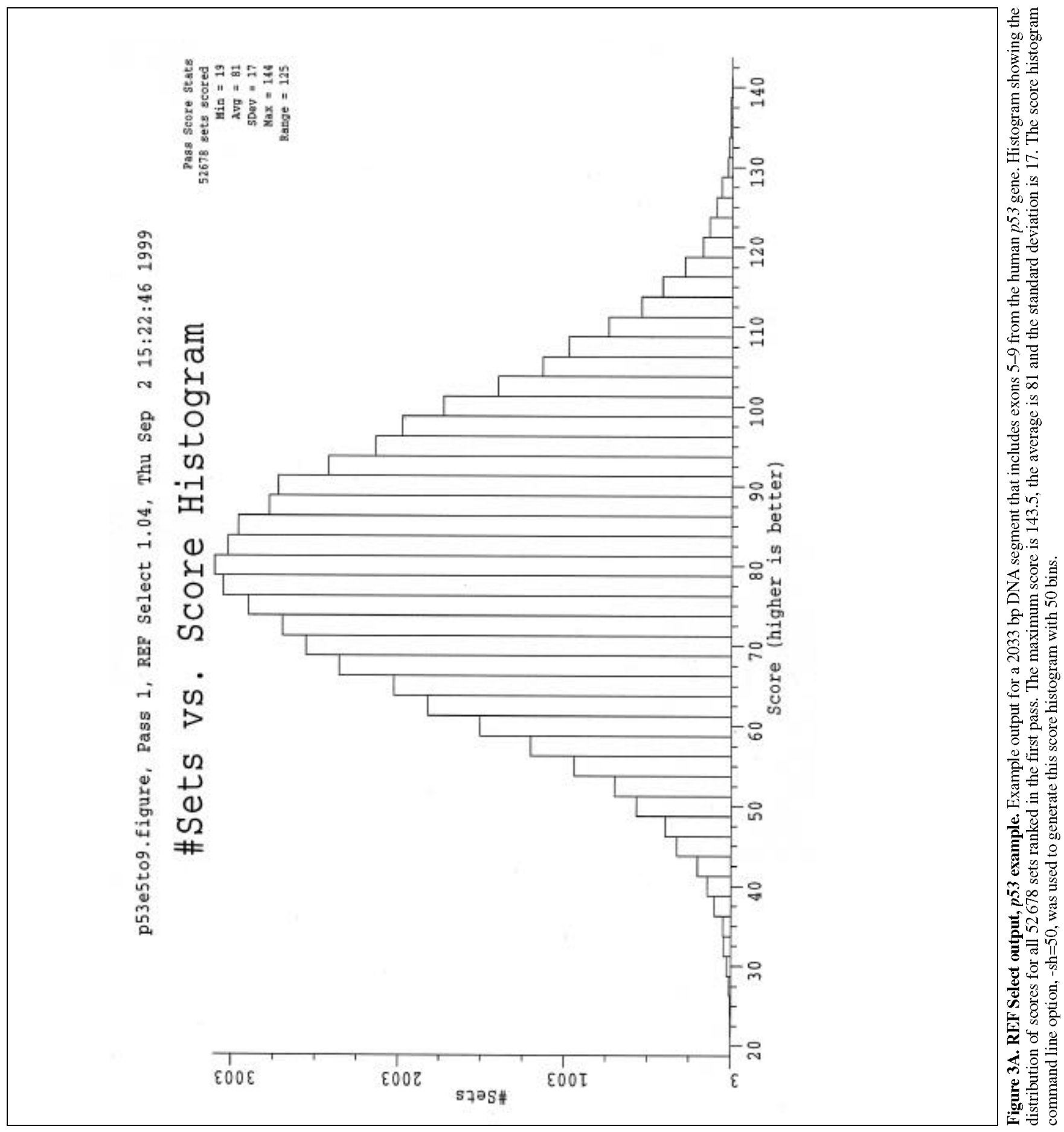




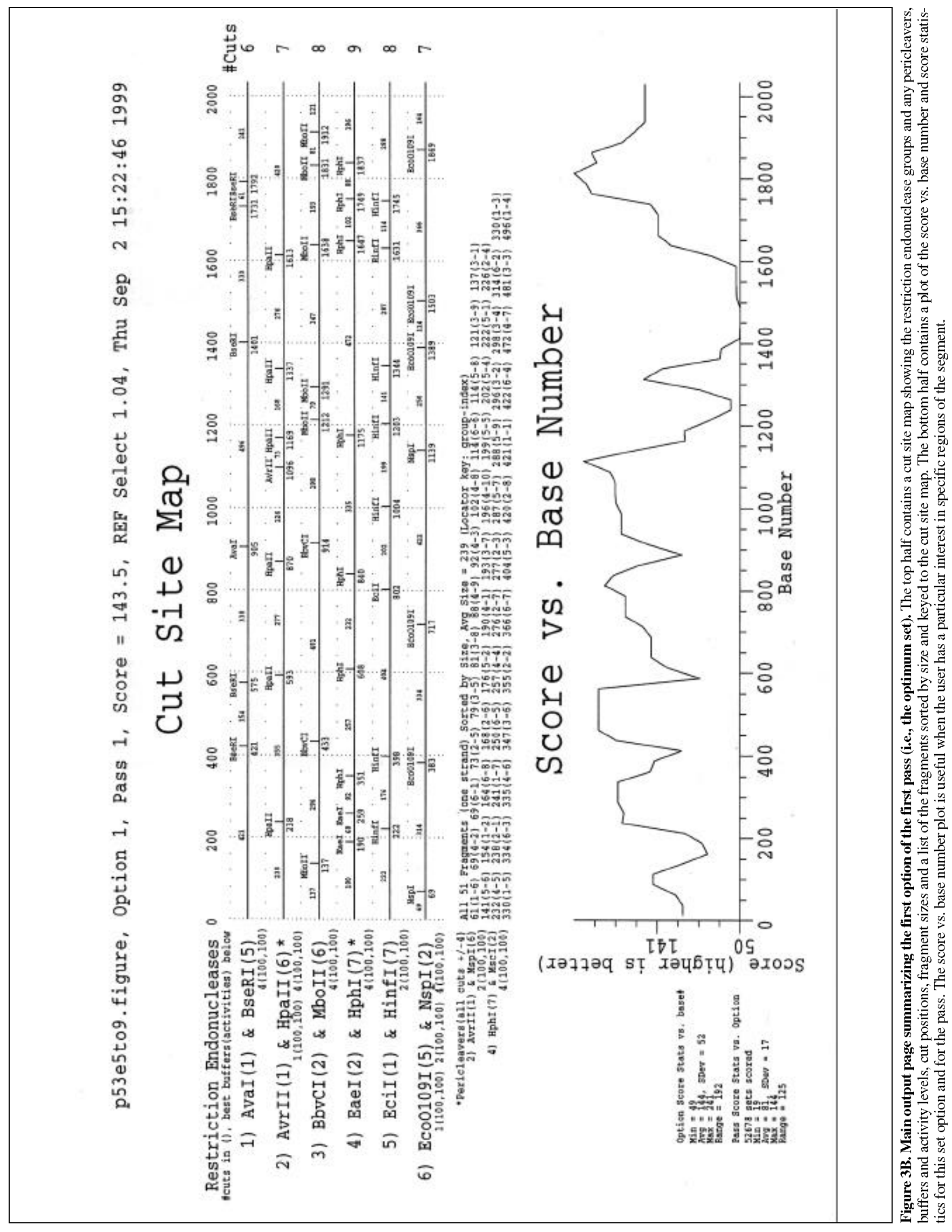


from group to group and the actual sequence differences in the nonoverlapping portions in future algorithms.

The size variety score for a particular base pair is defined in a way that it increases when the range of fragment sizes containing the base pair increases and when the fragments have a more uniform distribution over that range. Formally, the variety score $\mathrm{V}_{\mathrm{i}}$ for the $\mathrm{i}^{\text {th }}$ base pair is:

$V_{i}=\min \left[\left(s_{i, g}-s_{i, 1}\right), R_{s}\right]-\sum_{\mathrm{j}=2}^{\mathrm{g}}\left|\left(s_{i, j}-s_{i, j-1}\right)-\Delta_{i, a v g}\right|$

where $g$ is the number of groups in a REF set, $s_{i, 1}, s_{i, 2}, s_{i, 3}, \ldots$, $\mathrm{s}_{\mathrm{i}, \mathrm{g}}$ are the sizes of the fragments that contain the $\mathrm{i}^{\text {th }}$ base pair sorted by ascending size (one size representing both sense and antisense fragments), $\Delta_{i, a v g}=\left(\mathrm{s}_{i, g}-s_{i, 1}\right) /(\mathrm{g}-1)$ is the average fragment size delta for the $i^{\text {th }}$ base pair, min[] means take the minimum of the terms contained in the brackets, and $R_{s}$ is the fragment size range to use for scoring. The first term on the right side of Equation 1 adds to the variety score based on the range of fragment sizes. The second term subtracts from the variety score based on the deviation from a uniform distribution of fragment sizes. The overall score for a particular restriction endonuclease set is computed by averaging the individual base pair scores over the entire segment. With the default fragment size range constraint of 50-500 and the default $R_{s}$ of $75 \%$ of this range, the maximum possible score is 337.5. Typical scores are substantially lower than the maximum. Figure 1 shows a hypothetical example with a score of 131.3, and the highest scoring set for the $p 53$ example in the Appendix has a score of 143.5.

Using a value of $R_{s}$ that is somewhat less than the maximum possible fragment size range removes some of the bias towards the same range of fragment sizes for all regions of the segment. This bias may result in similar fragment sizes from different regions that may overlap on the gel. Detection sensitivity will not necessarily be compromised because only the fragment with the sequence change will be shifted, but the location of the change will likely be ambiguous. The value of $R_{s}$ represents a tradeoff between variety across fragments from the same region, which is critical for detection sensitivity and maximized when $R_{s}$ is $100 \%$ of the maximum possible range, and variety across fragments from different regions, which reduces the number of overlapping bands.

\section{Output and p53 Gene Example}

The Appendix shows an interactive run of REF Select for a p53 example using the default input parameter values. Figure $3 \mathrm{~A}$ shows the optional page of the figure output that shows the distribution of scores for the 52678 sets selected and ranked by REF Select in pass 1 . The distribution of scores is approximately normal, and the highest score of 143.5 is more than 3 standard deviations greater than the average score of 81. Figure $3 \mathrm{~B}$ shows the graphical summary page of the figure output for the highest scoring set (i.e., the optimum set). To implement the REF procedure using the optimum set of restriction endonucleases shown in Figure 3B, the amount of each restriction endonuclease to use must first be determined. Table 2 provides estimates that can be used as a starting point for digestion experiments to determine the optimum amounts.
In conclusion, REF Select is expert system software that assists in the selection of the set of groups of restriction endonucleases for restriction endonuclease fingerprinting. It enables a comprehensive review of the possible sets and a consistent, objective and fast selection of an optimal set for use in performing REF on a specific DNA segment. The default selection and ranking parameters used by REF Select were derived from our experience with the REF method and form a knowledge base that a user can customize and save. The user interface is simple and includes online help. The software generates both text and high-quality figure outputs.

\section{REFERENCES}

1.Broeks, A., A. de Klein, A.N. Floore, M. Muijtjens, W.J. Kleijer, N.G. Jaspers and L.J. van't Veer. 1998. ATM germline mutations in classical ataxia-telangiectasia patients in the Dutch population. Hum. Mutat. 12:330-337.

2.Du, Y.Z., A.K. Srivastava and C.E. Schwartz. 1998. Multiple exon screening using restriction endonuclease fingerprinting (REF): detection of six novel mutations in the L1 cell adhesion molecule (L1CAM) gene. Hum. Mutat. 11:222-230.

3.Feng, J., J.L. Sobell, L.L. Heston, E.H.J. Cook, D. Goldman and S.S. Sommer. 1998. Scanning of the dopamine D1 and D5 receptor genes by REF in neuropsychiatric patients reveals a novel missense change at a highly conserved amino acid. Am. J. Med. Genet. 81:172-178.

4.Feng, J., J.L. Sobell, L.L. Heston, D. Goldman, E.J. Cook, H.R. Kranzler, J. Gelernter and S.S. Sommer. 1998. Variants in the alpha2A AR adrenergic receptor gene in psychiatric patients. Am. J. Med. Genet. $81: 405-410$.

5.Hayashi, K. 1992. PCR-SSCP: a method for detection of mutations. Genet. Anal. Tech. Appl. 9:73-79.

6.Hayashi, K. and D.W. Yandell. 1993. How sensitive is PCR-SSCP? Hum. Mutat. 2:338-346.

7.Iwahana, H., K. Yoshimoto and M. Itakara. 1992. Detection of point mutations by SSCP of PCR-amplified DNA after endonuclease digestion. BioTechniques 12:64-66.

8.Liu, Q., J. Feng and S.S. Sommer. 1997. In a blinded analysis, restriction endonuclease fingerprinting detects all the mutations in a $1.9-\mathrm{kb}$ segment. BioTechniques 23:836-839.

9.Liu, Q. and S.S. Sommer. 1994. Parameters affecting the sensitivities of dideoxy fingerprinting and SSCP. PCR Methods Appl. 4:97-108.

10.Liu, Q. and S.S. Sommer. 1995. Restriction endonuclease fingerprinting (REF): a sensitive method for screening mutations in long, contiguous segments of DNA. BioTechniques 18:470-477.

11.Orita, M., H. Iwahana, H. Kanazawa, K. Hayashi and T. Sekiya. 1989. Detection of polymorphisms of human DNA by gel electrophoresis as single-strand conformation polymorphisms. Proc. Natl. Acad. Sci. USA 86:2766-2770.

12.Roberts, R.J. and D. Macelis. 1999. REBASE-restriction enzymes and methylases. Nucleic Acids Res 27:312-313.

13.Sommer, S.S. 1996. REF and ddF restriction endonuclease and dideoxy fingerprinting, p. 27-32. In U. Landegren (Ed.), Laboratory Protocols for Mutation Detection. Oxford Press, New York.

14.Stankovic, T., P. Weber, G. Stewart, T. Bedenham, J. Murray, P.J. Byrd, P.A. Moss and A.M. Taylor. 1999. Inactivation of ataxia telangiectasia mutated gene in B-cell chronic lymphocytic leukaemia [see comments]. Lancet 353:26-29.

Received 6 July 1999; accepted 20 September 1999.

Address correspondence to:

Dr. Steve S. Sommer

Director, Departments of Molecular Genetics and Molecular Diagnosis

City of Hope National Medical Center

1500 East Duarte Road

Duarte, CA 91010-3000, USA

Internet: ssommer@coh.org 
Appendix. REF Select Input Details, p53 Example

Shown here are the details of REF Select input that demonstrate the process of running the software for a sample $2033 \mathrm{bp}$ DNA segment that includes exons 5-9 from the human p53 gene. The following log shows how the GCG Mapsort file was generated for the example.

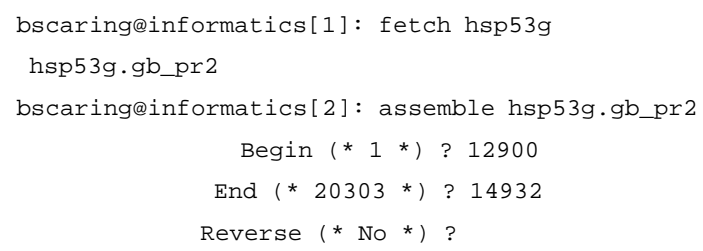

First, the command, Fetch, was used to get the entire human $p 53$ gene sequence file from the GenBank database. Then, the command, Assemble, was used to select the segment of interest and to renumber the segment to start from one. The Assemble step can be skipped if the segment is contiguous and renumbering the original sequence is not desired. Finally, the command, Mapsort, was used to find the restriction endonucleases that cut the segment and the corresponding cut sites. When using a restriction endonuclease property file that includes restriction endonucleases with isoschizomers, it is important to ensure that these restriction endonucleases are output by Mapsort, which, by default, lists only one of the isoschizomers. Specifically, the user must instruct Mapsort to output all isoschizomers by using ** in the enzyme field. The following is an excerpt showing only the first five restriction endnonucleases from the restriction endonuclease property file used in the example.

Restriction Endonucleases from the 1998/99 Catalog of New England Biolabs, Beverly, MA with the 6/10/1999 update of the NEBuffer Activity Chart from NEB's web site at http://www.neb.com/neb/tech/tech_resource/restriction/buffers/buffer_chart.html

\begin{tabular}{|c|c|c|c|c|c|c|c|}
\hline Name & Available & Desirable & Temp & Buf1 & Buf2 & Buf3 & Buf 4 \\
\hline AatII & $\mathrm{Y}$ & Y & 37 & 0 & 50 & 50 & 100 \\
\hline $\operatorname{AccI}$ & Y & Y & 37 & 50 & 50 & 10 & 100 \\
\hline Acc 65 I & Y & $\mathrm{Y}$ & 37 & 10 & 75 & 100 & 25 \\
\hline AciI & $\mathrm{Y}$ & $\mathrm{Y}$ & 37 & 25 & 50 & 100 & 50 \\
\hline Acl I & $\mathrm{Y}$ & $\mathrm{Y}$ & 37 & 10 & 10 & 0 & 100 \\
\hline
\end{tabular}

(file truncated for brevity)

The restriction endonuclease property file is an ASCII text file that contains a tab- or space-delimited table having columns for availability $(\mathrm{Y}$ or $\mathrm{N})$, desirability $(\mathrm{Y}$ or $\mathrm{N})$, optimum digestion temperature (Celsius) and buffer activity levels $(0-100)$ for specific buffers. Note that spaces must be removed from restriction endonuclease names when they are entered. The header of the example indicates the supplier from which the optimum digestion temperature and buffer activity level data were obtained, but does not imply that only this supplier can be used. The range of buffer activity levels allowed in the restriction endonuclease property file is $0-100$, which is consistent with activity levels normalized to a percentage of a maximum activity level. Any buffer activity levels provided as absolute values must be normalized by the maximum activity to get a percent value. If enzymes from multiple suppliers are used, then the user must devise the appropriate conversion and normalization scheme to get comparable activity levels.

The following is a log (edited for brevity) of the sample interactive run of REF Select using the Mapsort file generated as shown, the restriction endonuclease property file shown excerpted and the default input parameter values. 


\section{Appendix (cont.)}

bscaringeinformatics [4]: refsel p53e5to9. mapsort - sh=50

A score histogram will be computed with 50 bins.

Enter the format of the restriction site file p53e5to9.mapsort or enter "?" for help.

1. GCG Mapsort file.

2. TACG file with "Display Cut Sites by Enzyme" option. saved as a text file.

3. Webcutter 2 file with "Table of sites, sorted by enzyme name" option saved as a text file and edited to delete everything but this table.

$(* 1 *)$ :

Reading Mapsort file p53e5to9.mapsort...

1. Aat I cuts at $\mathrm{AGG}^{\prime} \mathrm{CCT} 2 \mathrm{x}: 3921032$

2. ACCI cuts at $\mathrm{GT}^{\prime} \mathrm{mk} \mathrm{AC} 1 \mathrm{x}: 16$

3. ACCII cuts at CG'CG 2x: 247253

(output of restriction endonuclease list truncated for brevity)

306 restriction endonucleases that cut segment read from file p53e5to9.mapsort

Use default restriction endonuclease property file named re_prop.txt ?

Hit return to use or

enter "c" to change to a different property file or

enter "?" for help or

enter " $n$ " to not use a property file:

194 restriction endonucleases read from property file re_prop.txt

193 were marked as available and desirable.

105 also cut the segment and will be used.

Use a previously saved parameter file?

Hit return for default parameter file refsel.par or

enter " $c$ " to change to a different parameter file or

enter " $n$ " to use default values:

Current Input Parameters

GROUP SELECTION CONSTRAINTS

1. Max optimal digestion temp difference within a group(C): 5

2. Min buffer activity levels: 100, 100

3. Fragment size range: $50-500$

4. Desired range of \#groups to select: $25-125$

SET SELECTION CONSTRAINTS

5. \#restriction endonuclease groups in a set: 6

6. Desired total \#fragments for all groups: $100(+10 \%,-20 \%)$

7. $5^{\prime}$ end frag max size(\#bp), limit(\#groups): 200,3

8. $3^{\prime}$ end frag max size(\#bp), limit(\#groups): 200, 3

9. Common cuts tolerance (\#bp), limit (\#cuts): 4, 2

SCORING ALGORITHM PARAMETERS

10. Scoring Algorithm Options: ㅇagment size range to use for scoring: 75

OUTPUT PARAMETERS

11. \#passes, \#groups to not use in next pass: 3,2

12. \#options to show for each pass: 3

13. Base pair range size for score plot: 25

14. Fragment size bin size: 50

15. Output text file line length, lines per page: 80,60

PROGRAM MODE

16. Auto parameter adjustment: ENABLED

Use these parameter values?

Hit return for yes or

enter item number for help or to change or

enter " $d$ " to use default values:

The output text file name will be p53e5to9.ref

Hit return to use or enter " $c$ " to change:

The output GCG figure file name will be p53e5to9.figure

Hit return to use or enter " $C$ " to change:

Selecting from $5.565000 e+03$ possible restriction endonuclease groups

which would form 4.114220 e+19 possible REF sets...

71 restriction endonuclease groups selected...

14 groups had pericleavers (all cut sites were identical within +/-4 bp).

Best pericleaver will be used and others will be listed as alternatives. 


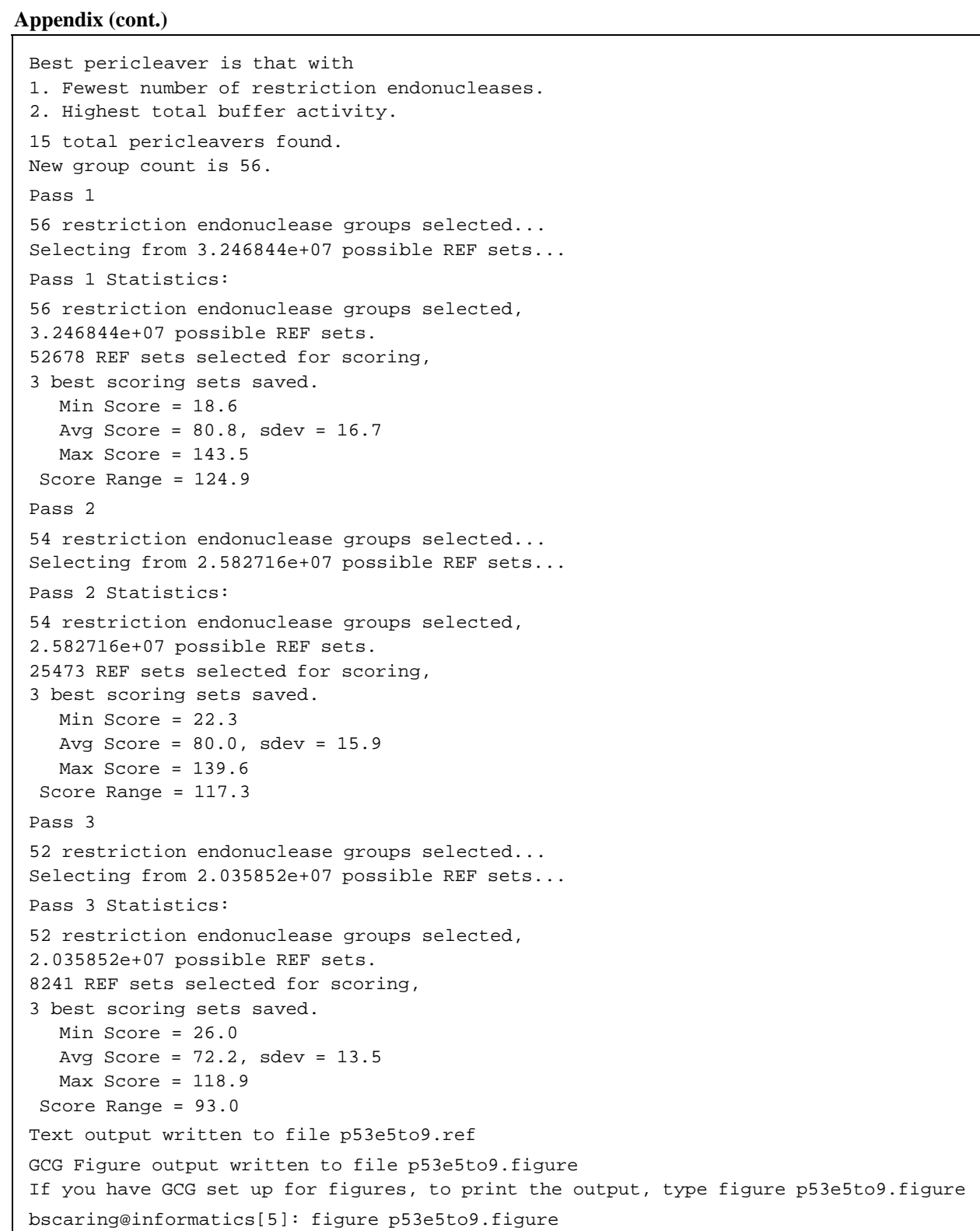

After reading the Mapsort file, REF Select indicated that it found 306 restriction endonucleases that cut the segment. Next, REF Select read the restriction endonuclease property file and found that it contained 193 restriction endonucleases marked as available and desirable, 105 of which cut the segment. From these 105 restriction endonucleases, 5565 groups of one or two can be formed and from those groups, $4 \times 10^{19}$ sets of six groups can be formed.

Proceeding with the selection step using the default parameter values, REF Select determined that 71 of the 5565 possible groups satisfied the group selection constraints. Of these 71 groups, 15 were found to be pericleavers of (to have the same cut sites as) other groups within a tolerance of $4 \mathrm{bp}$. Thus, 56 groups were selected in the first pass. Of the 32468436 possible sets of six groups that can be formed from these 56 groups, 52678 were found to satisfy the set selection constraints. In the ranking step, REF Select scored these 52678 sets and output the 3 best scoring sets and other run information. Figure 3B shows the output for the highest scoring set. The multiple pass feature was used in this example so that, in passes 2 and 3 , the two groups most often used in the previous pass were removed from consideration and that set selection proceeded by using the remaining 54 and 52 groups, respectively. 